\title{
REVIEW
}

\section{von Willebrand factor and its relevance to cardiovascular disorders}

\author{
Gregory Y H Lip, Andrew D Blann
}

\begin{abstract}
In the past decade the importance of the vascular endothelium in cardiovascular pathophysiology has become more apparent. One substance that is synthesised by and stored in endothelial cells is von Willebrand factor (vWF). When released, vWF seems to mediate platelet aggregation and adhesion to the vascular endothelium. Because the release of vWF is increased when endothelial cells are damaged, vWF has been proposed as an indicator of endothelial disturbance or dysfunction. The availability of such an index of endothelial dysfunction may have clinical value, because measurement of such a marker can be a non-invasive way of assisting in diagnosis or as an indicator of disease progression. The known association between vWF, thrombogenesis, and atherosclerotic vascular disease also suggests that high concentrations of VWF may be an indirect indicator of atherosclerosis and/or thrombosis. In addition, high vWF concentrations have prognostic implications in patients with ischaemic heart disease and peripheral vascular disease.
\end{abstract}

(Br Heart f 1995;74:580-583)

Keywords: von Willebrand factor (vWF); thrombogenesis; atherogenesis; endothelium

It has long been recognised that abnormalities in the blood vessel wall, blood flow, and blood constituents may increase the tendency for thrombus to form (Virchow's triad). However, it is now apparent that the process of thrombus formation (thrombogenesis) requires com-

University Department of Medicine, City Hospital, Birmingham G Y H Lip

University Department of Surgery, University

Hospital of South

Manchester,

Manchester

A D Blann

Correspondence to:

Dr G Y H Lip, University

Department of Medicine

City Hospital, Dudley Road,

Birmingham B18 7QH.

Accepted for publication

13 June 1995 plex interactions involving endothelial injury; platelet adherence, aggregation, and release; thrombin generation; and fibrin formation. ${ }^{1}$

We are increasingly aware that the vascular endothelium is involved in the production of many substances that are intimately associated with the regulation of vessel tone and permeability, haemostasis, fibrinolysis, and synthesis of growth factors. The release of substances inhibiting contraction (for example, endothelial derived relaxing factor or EDRF) and platelet activation are examples of protective mechanisms that maintain normal blood pressure and cardiovascular haemodynamics.

Another substance, von Willebrand factor (vWF), is synthesised by and stored in endothelial cells. It seems to be important in mediating platelet aggregation and adhesion to the vascular endothelium and subendothelium. ${ }^{2}$ Because vWF release increases when endothelial cells are damaged, the concentration of vWF has been proposed as an indicator of endothelial disturbance or dysfunction. ${ }^{3}$ The availability of such an index of endothelial dysfunction may have clinical value because measurement of such a marker can be a noninvasive way of assisting in diagnosis or as an indicator of disease progression (table 1). Endothelial dysfunction is, of course, important because this can change the ability of the cell to participate adequately in both coagulation and fibrinolysis; these changes may predispose to thrombus and atherosclerosis formation. ${ }^{13}$

\section{Ischaemic heart disease}

The association between vWF concentrations and ischaemic heart disease is well recognised. There is an association between vWF and the clinical severity of angina, indicating a role for endothelial dysfunction in the pathogenesis of coronary artery disease, and changes in vWF concentrations with acute myocardial infarction have been found. The mechanism for this release of VWF in acute myocardial infarction may be related to the generation of free radicals that follows myocardial infarction and

Table 1 vWF as a marker of endothelial dysfunction in vascular disorders

Associations between $v$ WF and the following cardiovascular disorders and risk factors have been reported:

Cardiovascular disorders

Ischaemic heart disease ${ }^{\star}$

- Peripheral vascular disease

- Left ventricular aneurysms

Atrial fibrillation

- Pulmonary vascular disease

- Cerebrovascular disease

- Deep venous thrombosis

Cardiovascular risk factors

- Diabetes

- Hypertension

-Homocysteinuria

- Hypercholesterolaemia

-Smoking

$\star_{v}$ WF may have prognostic implications in these conditions. 
reperfusion. ${ }^{4}$ Patients with angiographic patency after thrombolytic therapy with alteplase (tissue plasminogen activator, rt-PA) also demonstrate a fall in vWF within 24 hours of thrombolysis, when compared with patients with an occluded artery. ${ }^{5}$

An hour after inflation in percutaneous transluminal coronary angioplasty vWF increases, indicating endothelial injury (which is associated with increased free radical activity). ${ }^{6}$ This suggests endothelial disturbance after free radical generation which occurs with reperfusion during angioplasty.

vWF also seems to be an index of the increased risk of reinfarction and mortality in survivors of myocardial infarction. In a prospective follow up study of 123 survivors of myocardial infarction, high concentrations of vWF were independently associated with both reinfarction and death. ${ }^{7}$ Similarly, in the PLAT (Progetto Lombardo Atero-Tombosi) study, vWF concentrations were a significant predictor of atherothrombotic events in patients with angina pectoris. ${ }^{8}$ In a 16 year follow up study of 1393 men, Meade et al reported that increased concentrations of vWF were significantly associated with fatal ischaemic heart disease. ${ }^{9}$

The precise mechanism by which vWF increases risk in patients with ischaemic heart disease remains unknown. Because of the role of VWF in both adhesion and aggregation of platelets and in coagulation, the increased concentrations of $\mathrm{vWF}$ may increase the risk of thrombus formation in patients with preexisting disease of the vascular wall, such as coronary artery disease, and thus lead to further reinfarctions. Alternatively, increasing endothelial damage in coronary artery disease may result in increased thrombin generation, resulting in higher concentrations of vWF.

\section{Left ventricular dysfunction}

In a study of patients with ischaemic heart disease and cardiac dysfunction (as defined by radionuclide ventriculography) those with left ventricular aneurysms had the highest concentrations of plasma vWF. ${ }^{10}$ This may be explained by two possible mechanisms. Firstly, these patients seem to have the greatest endothelial dysfunction (as reflected by vWF concentrations), potentially leading to the most intravascular thrombogenesis (this is consistent with the high plasma concentrations of $\mathrm{D}$-dimer being found in these patients). Secondly, patients with the highest vWF concentrations have the greatest cardiovascular risk and consequently these patients may sustain the largest myocardial infarctions, or recurrent infarctions, ${ }^{7}$ resulting in the most cardiac "damage" and subsequent aneurysm formation.

Traditionally, heart failure has been regarded as being caused by abnormalities in the systolic or contractile function of the heart. However, $30-40 \%$ of patients with congestive heart failure have normal systolic function. Endothelial dysfunction may thus be related to abnormalities of diastolic function seen in ischaemic heart disease. However, in a Doppler echocardiographic study of 106 patients with ischaemic heart disease, there were no significant differences in vWF concentrations between patients with and without diastolic dysfunction. ${ }^{11}$

\section{Atrial fibrillation}

The risk of stroke and thromboembolism is increased in atrial fibrillation. In a cross sectional study of 85 patients with atrial fibrillation, plasma concentrations of vWF were appreciably increased and were not altered by treatment with aspirin or warfarin. ${ }^{12}$ Concentrations of vWF were independent of the underlying cause of atrial fibrillation. ${ }^{12}$ There was also a modest correlation between plasma vWF and fibrin D-dimer, which is an index of fibrin turnover and thrombogenesis. ${ }^{12}$ This correlation accords with the association between abnormalities of the blood vessel wall (thus, endothelial) and thrombus formation (as part of Virchow's triad).

\section{Peripheral vascular disease}

Concentrations of VWF are increased in patients with peripheral vascular disease. In the Edinburgh Artery Study, concentrations of vWF were significantly higher in 121 study cases than in matched controls. ${ }^{13}$

The increased concentrations of vWF in patients with claudication may be due to the cytotoxic products of activated neutrophils. ${ }^{14}$ This increase in vWF was associated with a consumption of the antioxidant glutathione peroxidase, implying the possible role of cytotoxic reactive oxygen species. Further evidence of the possible role for these toxic species is provided by the inverse relation between vWF and glutathione peroxidase in patients with hypercholesterolaemia and/or concomitant vascular disease. ${ }^{14}$

vWF also has prognostic value in patients with peripheral vascular disease. For example, increased concentrations of vWF predict poor outcome of infrainguinal bypass grafting. ${ }^{15}$ In a one year follow up of 617 patients with claudication, an increased vWF concentration increased the risk of a coronary event by $1 \cdot 3,{ }^{16}$ but not as much as increases in cross linked fibrin degradation products $(2 \cdot 6)$ and plasma viscosity $(1 \cdot 8) .{ }^{16}$

\section{Pulmonary vascular disease}

The pulmonary vasculature also seems to influence plasma vWF concentrations. For example, in primary pulmonary hypertension the pulmonary endothelial cells show evidence of injury, and increases in plasma vWF have been shown in this condition. ${ }^{17}$ Increased plasma vWF was also found in patients with increased pulmonary vascular resistance and decreased cardiac output, irrespective of the presence of mitral stenosis. ${ }^{18}$ These findings accord with a haemodynamically induced increase in the endothelial release of VWF. 


\section{Hypertension}

Concentrations of vWF were significantly increased in patients with hypertension and normal in patients in whom hypertension was successfully treated. ${ }^{19}$ Microalbuminuria (defined as the excretion of urine albumin of between 20 and $200 \mu \mathrm{g} / \mathrm{min}$ ) and endothelial dysfunction also co-exist, suggesting that microalbuminuria reflects systemic dysfunction of the vascular endothelium. ${ }^{20}$ In hypertensive patients vWF concentrations were higher in those with microalbuminuria than in those without or in controls. ${ }^{20}$

Varizi et al also reported high vWF concentrations in hypertensive patients, which were related to diastolic blood pressure; there was also a modest correlation with the thickness of the left ventricular posterior wall and ventricular septum, and with the left ventricular mass index. ${ }^{21}$ This is important because the presence of left ventricular hypertrophy is another index of hypertensive target-organ damage, and such patients are at high risk of cardiovascular events.

Mean vWF concentrations were significantly higher in women with pregnancyinduced hypertension and pre-eclampsia than in healthy pregnant women, and concentrations correlated with the severity of the condition. $^{22}$ This may indicate a role in the microangiopathy observed in the disease.

Clearly, hypertension can promote endothelial dysfunction or damage, but it has been suggested that endothelial damage can promote hypertension. ${ }^{23}$ In addition, the presence of such endothelial damage may be one mechanism by which patients with hypertension are at risk of thromboembolism. Whether

\section{Table 2 vWF concentrations in cardiovascular disease: cause or consequence?}

vWF as a likely "cause" of cardiovascular disease

-vWF concentrations have a hereditary influence

- High vWF concentrations are found after endothelial injury by smoking, hypertension or hyperlipidaemia

- Association between vWF with the clinical severity of angin

- Raised plasma vWF concentrations preceed cardiovascular events-for example, in predicting reinfarction after myocardial infarction ${ }^{7}$ and in patients with peripheral vascular disease ${ }^{15} 16$ -In patients with inflammatory vascular disease, high vWF concentrations predict mortality, disease progression, and cardiovascular events ${ }^{28}$

-Increases in plasma vWF concentrations in many cardiovascular disorders and atherosclerosis risk factors are not always associated with an active acute phase response

vWF as a "consequence" of cardiovascular disease

-vWF concentrations may be increased by glucocorticoids and cytokines such as interleukin-1 and tumour necrosis factor (TNF) which are produced by monocytes and macrophages and tumour necrosis factor (TNF) which are produced by monocytes and macrophages Increased vWF concentrations may simply reflect endothelial activation or stimulation and
not endothelial dysfunction. Thus occlusive disease of arteries by (for example) thrombus may not endothelial dysfunction. Thus occlusive disease
cause a reactive increase in $\mathrm{VWF}$ in the circulation.

The precise mechanism for the increase in vWF in cardiovascular disorders remains uncertain, although a cytokine-mediated increase in synthesis is likely to be the common pathway.

\section{Table 3 Measures suggested in modulating concentrations of von Willebrand factor}

- Control of risk factors, including obesity, may reduce vWF concentrations

- Lower concentrations of VWF may be obtained in diabetics who are able to control their ketoacidosis

- Patients with coronary atherosclerosis who had been on a strict diet to reduce their cholesterol had lower concentrations of $\mathrm{VWF}$ than a similar cohort who were not intensively dieted - Conventional lipid lowering treatment also lowered vWF in hypercholesterolaemic patients with and without symptomatic vascular disease

- Abstention from smoking and control of hypertension reduces vWF

- (Possibly) the use of hormone replacement therapy (HRT) in women with premature menopause

The central role of vWF in thrombogenesis and atherogenesis has made it a target for research into antithrombotic treatments centred on antibodies, peptides, or other compounds that inhibit vWF function that have been suggested as new anticoagulants. or not this relation also extends to the associated complications of hypertension remains to be seen.

\section{Cerebrovascular disease}

High concentrations of VWF are associated with ischaemic cerebrovascular disease. ${ }^{2425}$ Patients with thrombotic strokes have higher vWF concentrations than those with haemorrhagic strokes. ${ }^{25}$ This association between vWF and cerebral thrombosis may reflect either endothelial dysfunction associated with cerebral thrombosis (or its risk factors, such as hypertension) or ischaemia related release of vWF from infarcted tissue. The precise mechanism, however, remains unknown.

\section{Deep venous thrombosis}

High concentrations of vWF have been associated with an increased incidence of thromboembolic events. For example, early work reported an association between vWF concentrations and deep venous thrombosis. ${ }^{26}$ The prospective value of vWF was also demonstrated in a study of patients after major abdominal surgery where high preoperative concentrations of vWF were associated with an increased risk of postoperative deep venous thrombosis. ${ }^{27}$

\section{Modulating vWF?}

Because there is compelling evidence that high concentrations of vWF are to be avoided, methods should be sought to reduce to reduce the production of vWF. There is also debate whether the high vWF concentrations seen in cardiovascular disorders are the cause or consequence of the disease process (table 2).

vWF does not seem to be affected by conventional protocols for non-pharmacological cardiovascular intervention. Methods to reduce $\mathrm{VWF}$ thus remain experimental, and as yet there are no definitive methods of therapeutically altering plasma vWF concentrations. In addition, there is no evidence that measures to reduce circulating vWF will have beneficial effects. However, because vWF is increased in each of the four major risk factors for atherosclerosis (hypertension, hypercholesterolaemia, smoking, and diabetes) then this is perhaps one area where progress may be made (table 3).

\section{Conclusion}

Much has been learnt about the cellular and molecular biology of vWF since the distinction was made between this protein and factor VIII nearly 20 years ago. Many case-control studies support the hypothesis that high concentrations of vWF are an index of atherosclerosis or of an increased risk of thrombogenesis or both. von Willebrand factor may therefore be a good marker for endothelial dysfunction in various diseases. Furthermore, it may be involved in the disease process by making a considerable contribution towards thrombogenesis and 
atherogenesis via platelet adhesion and aggregation.

We acknowledge the assistance of the British Heart Foundation and the British Society for Thrombosis and Haemostasis in our and the British Society for Thrombosis and Haemostasis in our research work. GL is recipient of the 1994 Edith Walsh and Ivy
Powell Research Awards for cardiovascular research from the British Medical Association.

1 Badimon L, Badimon JJ, Fuster V. Pathogenesis of thrombosis. In: Fuster V, Verstraete $M$, eds. Thrombosis in cardiovascular disorders. Philadelphia: WB Saunders, 1992:17-39.

2 Boneu B, Abbai M, Plante J, Bierme R. Factor VIII complex and endothelial damage. Lancet $1975 ; \mathbf{i}: 1430$.

3 Blann AD. von Willebrand factor and the endothelium in vascular disease. Br ₹ Biomed Sci 1993;50:125-34.

4 Davies SW, Ranjadayalan K, Wickens DG, Dormandy TL, Timmis AD. Lipid peroxidation associated with successful thrombolysis. Lancet 1990;335:741-3.

5 Andreotti F, Hackett DR, Haider AW, Roncaglioni MC, Davies GJ, Beacham JL, et al. von Willebrand factor, plasminogen activator inhibitor-1 and C-reactive protein plasminogen activator inhibitor-1 and C-reactive protein
are markers of thrombolytic efficacy in acute myocardial are markers of thrombolytic efficacy in acute m

6 Blann A, Midgley H, Burrows G, et al. Free radicals, antioxidants and endothelial cell damage after percutaneous transluminal coronary angioplasty. Coron Artery Dis 1993;4:905-10.

7 Jansson J-H, Nilsson TK, Johnson O. von Willebrand factor in plasma: a novel risk factor for recurrent myocardial infarction and death. Br Heart $\mathcal{F} 1991 ; 66: 351-5$.

8 Cortellaro $M$, Boschetti C, Cofrancesco $E$, et al. The PLAT study: hemostatic function in relation to atherothrombotic ischemic events in vascular disease patients. Arteriosclerosis Thrombosis 1992;12:1063-70.

9 Meade TW, Cooper JA, Stirling Y, Howarth DJ, Ruddock V, Miller GJ. Factor VIII, ABO blood group and the incidence of ischaemic heart disease. $\mathrm{Br} f$ Haematol dence of ischa

10 Lip GYH, Lowe GDO, Metcalfe MJ, Rumley A, Dunn FG. Effect of warfarin therapy on plasma fibrinogen, von Willebrand factor and fibrin D-dimer in left ventricular dysfunction secondary to coronary artery disease with and without aneurysms. Am $\mathcal{F}$ Cardiol 1995;76:453-8.

11 Lip GYH, Lowe GDO, Metcalfe MJ, Rumley A, Dunn FG. Is diastolic dysfunction associated with thrombogenesis? A study of circulating markers of a prothrombotic state in patients with coronary artery disease. Int $f$ Cardiol 1995;50:31-42.

12 Lip GYH, Lowe GDO, Rumley A, Dunn FG. Increased markers of thrombogenesis in chronic atrial fibrillation:

Effects of warfarin therapy. Br Heart f 1995;73:527-33.

13 Smith FB, Lowe GDO, Fowkes FG, et al. Smoking, hemo- static factors and lipid peroxides in a population casecontrol study of peripheral arterial disease. Atherosclerosis 1993;102:155-62.

14 Blann AD, Maxwell SRI, Burrows G, Miller JP. Antioxidants, von Willebrand factor and endothelial cell damage in hypercholesterolaemia and vascular disease. Atherosclerosis 1995;116:191-8.

15 Woodburn KF, Rumley A, Pollock JG, Quin RO, Lowe GD. Prediction of outcome in infrainguinal bypass grafting. Br f Surg 1993;80:525-6.

16 Fowkes FGR, Lowe GDO, Housley E, Rattray A, Rumley A, Elton RA, et al. Cross linked fibrin degradation products, progression of peripheral arterial disease, and risk of coronary artery disease. Lancet 1993;342: 84-6.

17 Geggel RL, Carvalho AC, Hoyer LW, Reid LM. von Willebrand factor abnormalities in primary pulmonary hypertension. Am Rev Respir Dis 1987;135:294-9.

18 Penny WF, Weinstein M, Salzman EW, Ware JA. Correlation of circulating von Willebrand factor levels with cardiovascular hemodynamics. Circulation 1991;83:1630-6.

19 Blann AD, Naqvi T, Waite M, McCollum CN. von Willebrand factor and endothelial damage in essential hypertension. $\mathcal{F}$ Hum Hypertens 1993;7:107-11.

20 Pedrinelli R, Giampietro O, Carmassi F, et al. Microalbuminuria and endothelial dysfunction in essential albuminuria and endothelial dysfunct

21 Varizi ND, Smith DH, Winer RL, Weber MA, Gonzales EC, Meutel JM. Coagulation and inhibitory and fibrinolytic proteins in essential hypertension. $₹ \mathrm{Am} \mathrm{Soc}$ Nephrol 1993;4:222-8.

22 Deng L, Bremme K, Hansson LO, Blomback M. Plasma levels of von Willebrand factor and fibronectin as markers of persisting endothelial damage in preeclampsia. Obstet Gynecol 1994;84:941-5.

23 Luscher TF. The endothelium: Target or promoter of hypertension? Hypertension 1990;15:482-5.

24 Uchiyama S, Takeuchi $M$, Osawa $M$, Kobayashi I, Maruyama S, Aosaki M, Hirosawa K. Platelet function tests in thrombotic cerebrovascular disorders. Stroke tests in throm

$25 \mathrm{Liu} \mathrm{L}$, Lin Z, Shen S. Changes of von Willebrand factor and antithrombin III levels in acute stroke: Differences between thrombotic and haemorrhagic stroke. Thromb Res 1993;72:353-8.

26 Wahlberg T, Blomback M, Overmark I. Blood coagulation studies in 45 patients with ischemic cerebrovascular disease and 44 patients with venous thromboembolic disease. Acta Med Scand 1980;207:385-90.

27 Nilsson T, Mellbring G, Hedner U. Relationship between factor XII, von Willebrand factor and postoperative deep venous thrombosis. Acta Chir Scand 1986;152:347-9.

28 Blann $\mathrm{AD}$. von Willebrand factor as a marker of injury to the endothelium in inflammatory vascular disease. $\mathcal{F}$ Rheumatol 1993;20:1469-71. 